\title{
Producción de Sílice Mesoporosa Empleando Monoestearato de Glicerol como Porógeno Oleoquímico
}

\author{
Juan D. Peña, Eliana M. Cardona, Juan M. Marín y Luis A. Rios \\ Universidad de Antioquia, Facultad de Ingeniería, Grupo de Procesos Fisicoquímicos Aplicados, \\ Apdo. .Aéreo 1226, Medellín-Colombia (e-mail: larios@udea.edu.co)
}

\begin{abstract}
Resumen
El presente trabajo describe el desarrollo de una novedosa síntesis de sílices mesoporosas basada en un mecanismo en dos etapas en medio ácido $(\mathrm{pH}=2)$, empleando materias primas renovables. La primera etapa consistió en la formación de micelas híbridas estables, producto de la interacción entre silicatos y el agente porógeno, y la segunda en la policondensación de silicatos para formar sílice. Se evaluaron dos fuentes de silicio: tetraetilortosilicato (TEOS) y metasilicato de sodio (MSiNa). Como porógeno oleoquímico se usó monoestearato de glicerol y como iniciador de la reacción de policondensación se usó fluoruro de sodio (NaF). Las sílices obtenidas con las mejores propiedades texturales, poseen características superficiales interesantes para un potencial aplicación en catálisis heterogénea, con área superficial BET de $727.4 \mathrm{~m}^{2} / \mathrm{g}$, volumen total de poros de $0.50 \mathrm{~cm}^{3} / \mathrm{g}$ y distribución de tamaños de poro alrededor de $46.2 \AA$.
\end{abstract}

Palabras clave: metasilicato de sodio, monoestearato de glicerol, porógeno oleoquímico, sílices mesoporosas, tetraetilortosilicato.

\section{Production of Mesoporous Silica using Glycerol Monostearate as Oleochemical Porogen}

\begin{abstract}
This paper describes the development of a novel synthesis of mesoporous silica based on a twostep process, in acidic media $(\mathrm{pH}=2)$, using renewable raw materials. The first stage was the formation of stable hybrid micelles, product of the interaction between the porogen and silicates, and the second was the polycondensation of silicates to produce silica. Two sources of silicon were used: tetraethylortosilicate (TEOS) and sodium metasilicate (MSiNa). Glycerol monostearate was used as oleochemical porogen and sodium fluoride was used as initiator for the reaction of condensation of silica. The silica obtained with the best textural properties have interesting surface characteristics for potential applications in heterogeneous catalysis, with BET surface area of 727.4 $\mathrm{m}^{2} / \mathrm{g}$, total volume of pores of $0.50 \mathrm{~cm}^{3} / \mathrm{g}$ and pore size distribution of about $46.2 \AA$.
\end{abstract}

Keywords: glycerol monostearate, mesoporous silica, oleochemical porogen, sodium metasilicate, tetraethylortosilicate 


\section{INTRODUCCIÓN}

La sílice es un material de naturaleza inorgánica $\left(\mathrm{SiO}_{2}\right)$ que resulta de la policondensación de los grupos silanol $(-\mathrm{Si}-\mathrm{OH})$ del ácido silícico $\left(\mathrm{Si}(\mathrm{OH})_{4}\right)$. Durante su formación, el precursor de silicio puede someterse a interacción con agentes porógenos o tensoactivos cuando se adicionan al medio de reacción. Estas sustancias poseen la facultad de formar micelas que durante la reacción de condensación son atrapadas por la creciente red inorgánica de siloxanos. Como resultado, se obtiene un material híbrido cuyo esqueleto orgánico, formado por las micelas de tensoactivo, puede ser removido por extracción con solventes o calcinación para dar origen a una estructura sólida basada en un arreglo regular o irregular de canales definidos por paredes de sílice (Sierra et al., 2000; Aramendia et al., 2004; Prouzet y Boissière, 2005; Liu et al., 2007; Mesa et al., 2007; Guth et al., 2007; Mesa et al., 2008; Wang y Shantz, 2008).

Gracias a su naturaleza química, las sílices son ampliamente usadas en procesos de separación y adsorción de moléculas, biomedicina y encapsulación, ensambles moleculares, cromatografía líquida de alta resolución (HPLC) y cromatografía de fluidos supercríticos, aditivos en pinturas, cargas reforzantes en caucho sintético, tecnología de membranas e ingeniería molecular, electrónica y óptica, tratamiento de superficies químico-mecánico (acabado espejo), y una aplicación industrial muy importante es su uso como soporte de catalizadores químicos o biológicos para formar catalizadores heterogéneos. Para todas estas aplicaciones se requiere que la sílice presente altas área superficial y porosidad, está última propiedad debe ser del tipo meso- y macro-porosa; además, para algunas de estas aplicaciones la sílice debe tener una morfología de partícula específica (Sierra et al., 2000; Vacassy et al., 2000; Biswas et al., 2007; Meynen et al., 2007; Sierra et al., 2008).

La formación de meso- y macro-porosidad en la sílice se logra con la adición de uno o varios tensoactivos en el medio de condensación. Los tensoactivos de uso convencional son de naturaleza diversa: iónicos, aniónicos, aminas, entre otros, y su selección depende de las características morfológicas logradas por las micelas formadas durante la reacción para obtener una estructura porosa deseada. Estudios recientes muestran las ventajas de usar tensoactivos no iónicos (neutros), con relación a tensoactivos que involucran interacciones electrostáticas, tales como su fácil remoción mediante extracción con solventes no corrosivos o calcinación, y la tendencia de los tensoactivos neutros a producir estructuras con paredes más gruesas y sólidos de menor tamaño de partícula, lo cual mejora la estabilidad y la textura porosa (Leonard et al., 2003; Aramendia et al., 2004; Prouzet and Boissière, 2005; Biswas et al., 2008).

Para la síntesis de sílices porosas se han utilizado tradicionalmente tensoactivos no iónicos tales como Triton X100, Pluronic F127, Twin 20, 40, 60 entre otros (Sierra et al., 2000; Aramendia et al., 2004; Guth et al., 2007; Mesa et al., 2007; Venkatathri, 2007; Mesa et al., 2008; Sierra et al., 2008), con los cuales se han obtenido sílices de altas áreas superficiales BET, estrechas distribuciones de tamaños de poros, morfologías y tamaños de partícula regulares. A pesar de esto, dichos tensoactivos presentan desventajas que restringen su aplicación a nivel industrial, como son, sus altos costos, debido a que se derivan de materias primas no renovables, y la necesidad de importación ya que ninguno es producido localmente. Considerando las ventajas de los tensoactivos no iónicos y la tendencia mundial para su uso en síntesis de sílices, en este trabajo se evaluó el uso de monoestearato de glicerol como agente porógeno, el cual es un producto de carácter renovable, no tóxico, de alta disponibilidad en el mercado local y de bajo precio.

El mono-estearato de glicerol, $\mathrm{CH}_{3}\left(\mathrm{CH}_{2}\right)_{16} \mathrm{COOCH}_{2} \mathrm{CCH}_{2} \mathrm{OHHOH}$, es el mono-ester de glicerol proveniente del ácido esteárico y la glicerina, físicamente se percibe como una pasta cremosa blanca, levemente soluble en agua caliente, soluble en alcohol, cloroformo, éter, y éter de petróleo, también es soluble en aceite. Posee un balance hidrófilo-lipófilo (HLB) de 3.8 por lo cual tiene buenas propiedades como antiespumante y es moderadamente soluble en agua. En la figura 1 se presentan la estructura molecular del monoestearato de glicerol (Chemical land 21, 2006).

El objetivo principal de este trabajo consiste en presentar el desarrollo de un nuevo proceso de síntesis de sílices mesoporosas que involucra el uso de monoestearato de glicerol como agente porógeno, un tensoactivo que hasta el momento no ha sido reportado para este tipo aplicaciones. 
Como materias primas se empleó TEOS y MSiNa como precursores de silicio, y NaF como iniciador de la reacción de condensación. Mediante análisis de fisisorción de nitrógeno y SEM, se determinaron las características porosas y morfológicas de los materiales obtenidos.

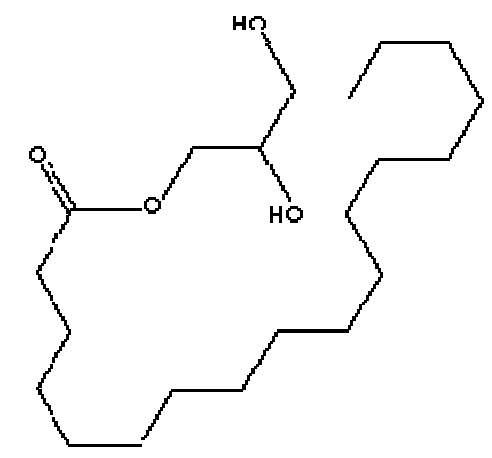

Fig. 1: Estructura molecular del Mono-estearato

\section{DESCRIPCIÓN EXPERIMENTAL}

El procedimiento de síntesis desarrollado se llevo a cabo en dos etapas, la primera consistió en la formación de micelas hibridas generadas por la interacción entre el porógeno y los silicatos (Si) disueltos en el medio de reacción; la segunda se basó en la reacción de condensación de sílice inducida por la adición de la sal NaF (Fluoruro de sodio al 99\%, Merck). Para esto, se prepararon dos soluciones en contenedores independientes, en uno se disolvió el precursor de silicio (tetraetilortosilicato TEOS 98\%, Merck o metasilicato de sodio nonahidratado, cristal 99\%, J.T. Baker) a $\mathrm{pH}$ ácido ajustado con una solución de ácido clorhídrico $1 \mathrm{M}$ a un valor de 2 . En el otro contenedor se disolvió el monoestearato de glicerol comercial en etanol a $60^{\circ} \mathrm{C}$ y bajo agitación magnética suave. La solución de silicatos fue calentada a $60^{\circ} \mathrm{C}$ y adicionada lentamente a la solución alcohólica de monoestearato de glicerol. El volumen se ajusto para obtener una relación volumétrica 1:1 etanolagua y posteriormente se adicionó la sal $\mathrm{NaF}$ para dar inicio a la reacción. La mezcla fue sometida a tratamiento térmico por tres días a $85^{\circ} \mathrm{C}$. Finalizada esta etapa, el material fue dispersado mediante ultrasonido, filtrado, lavado con etanol y secado a $60^{\circ} \mathrm{C}$ por $12 \mathrm{~h}$.

El tensoactivo ligado a la estructura de la sílice fue removido por calcinación en aire a $550^{\circ} \mathrm{C}$ con la siguiente rampa de calentamiento: $25^{\circ} \mathrm{C}-100^{\circ} \mathrm{C}$ con calentamiento de $1.5^{\circ} \mathrm{C} / \mathrm{min}, 100^{\circ} \mathrm{C}$ por 1 hora, $100^{\circ} \mathrm{C}-550^{\circ} \mathrm{C}$ con calentamiento de $1.5^{\circ} \mathrm{C} / \mathrm{min}$ y $550^{\circ} \mathrm{C}$ por 4 horas.

Para el diseño experimental sólo fueron consideradas las variables dominantes del proceso que son, el tipo de precursor de silicio y la relación molar Si:porógeno. El NaF es un iniciador de la reacción de policondensación de sílice ampliamente reportado en la literatura, cuyo efecto en la cinética de policondensación y características de materiales silíceos meso-estructurados ya ha sido estudiado (Prouzet y Boissière, 2005; Biswas, 2007; Jin et al., 2007a; Jin et al., 2007b). Fue de interés en esta investigación el manejo de cinéticas de reacción lentas que proporcionaran el tiempo requerido para una adecuada interacción entre el porógeno y los silicatos. Según los reportes, esto se logra con bajas concentraciones de $\mathrm{NaF}$ que incrementen sutilmente la fortaleza iónica del medio, sin afectar de forma significativa la meso-estructura del material (Prouzet y Boissière, 2005; Biswas, 2007; Jin et al., 2007-A; Jin et al., 2007-B). Por tal razón, la concentración de NaF no fue considerada una variable dominante del proceso, solo se determinó experimentalmente la cantidad de sal mínima requerida para dar inicio a la reacción bajo las distintas condiciones síntesis estudiadas.

El número de experimentos realizados corresponde a un diseño experimental tipo factorial, con dos factores evaluados a tres niveles. Los factores corresponden al tipo de precursor de silicio empleado y los niveles a las relaciones molares Si:porógeno evaluadas. Los valores asignados a estos niveles corresponden a los rangos normalmente reportados en la literatura y los límites de solubilidad del porógeno. Las relaciones molares evaluadas y la codificación de los materiales resultantes se muestran en la tabla 1. 
Tabla 1: Condiciones de síntesis

\begin{tabular}{cccc}
\hline \multirow{2}{*}{ Identificación } & Iniciador NaF requerido [g] & \multicolumn{2}{c}{ Relación molar Si:Porógeno } \\
\cline { 3 - 4 } & & TEOS & MSiNa \\
\hline TEG1 & 0,168 & 5 & - \\
TEG2 & 0,168 & 10 & - \\
TEG3 & 0,126 & 15 & - \\
B-TEOS & 0,42 & Sin Porógeno & - \\
MEG1 & 0,042 & - & 5 \\
MEG2 & 0,084 & - & 10 \\
MEG3 & 0,126 & - & 15 \\
B-MSiNa & 0,315 & - & Sin Porógeno \\
\hline
\end{tabular}

Para determinar el efecto del tensoactivo y el tipo de precursor de silicio empleado sobre las características porosas de las sílices resultantes, se uso un equipo para análisis de fisisorción MICROMERITICS GEMINI V que permite medir isotermas de adsorción-desorción de nitrógeno sobre materiales adsorbentes. Las muestras después ser calcinadas, fueron tratadas con un gas inerte (nitrógeno) a $100^{\circ} \mathrm{C}$ por una hora y luego con vacío a $200^{\circ} \mathrm{C}$ por dos horas. Las isotermas generadas por el equipo permitieron determinar el área superficial usando el modelo BET en un intervalo de presión relativa que va desde 0.05 hasta 3.5 , la distribución de tamaños de poro entre 1.7 y $20 \mathrm{~nm}$ por el método BJH y el tamaño medio de poros que corresponde al máximo de la curva de distribución de tamaño de poro. El volumen de los mesoporos fue determinado por el método BJH disponible en el software del equipo. Las características morfológicas y tamaño de partícula fueron determinadas con un microscopio electrónico (SEM) JEOL JSM 5910LV.

\section{RESULTADOS Y DISCUSIÓN}

Con base en las cantidades de NaF reportadas en la Tabla 1, puede afirmarse que el precursor de silicio tiene una fuerte influencia sobre el proceso de síntesis, la cual afecta principalmente la cinética de condensación de la sílice. Esta variable es más alta cuando se usa metasilicato de sodio con relación al tetraetilortosilicato (TEOS). Si se comparan las cantidades requeridas para las síntesis con TEOS con sus equivalentes empleando MSiNa puede verse que las primeras consumen mayor cantidad de $\mathrm{NaF}$, a excepción de la preparación TEG3 en la cual se adiciona la misma cantidad de $\mathrm{NaF}$ que en su equivalente MEG3. Este efecto en TEG3 se debe a la concentración de precursor de silicio, otra variable dominante en la cinética de condensación de sílice, que en este caso es bastante alta comparada con las preparaciones TEG1 y TEG2. El efecto del MSiNa en la cinética de reacción se atribuye a la interacción de los electrolitos disueltos en el medio que incrementan la fuerza iónica de la solución; estos electrolitos provienen de dos fuentes, los cationes sódicos $\left(\mathrm{Na}^{+}\right)$que entran acompañando al precursor de silicio $\left(\mathrm{Na}_{2} \mathrm{O} . \mathrm{SiO}_{2}\right)$ y los aniones cloruros $\left(\mathrm{Cl}^{-}\right)$que son introducidos durante la neutralización con $\mathrm{HCl}$ en el paso de medio alcalino a medio fuertemente ácido. La presencia de electrolitos en el medio de reacción favorece la deslocalización electrónica de los óxidos de silicio disueltos, fenómeno requerido para la interacción con los agentes porógenos no iónicos, y la asociación de las especies inorgánicas mediante enlaces siloxanos (-Si-O-Si-).

En cuanto a las características morfológicas de las sílices, el uso de monoestearato de glicerol como agente porógeno conduce a la formación de sólidos completamente amorfos, compuestos de aglomerados de partículas sin forma regular.

En la Figura 2 se muestran las micrografías SEM de las sílices TEG2, producida con TEOS, MEG2, producida $\mathrm{MSiNa}$, y $\mathrm{B}-\mathrm{MSiNa}$, producida con $\mathrm{MSiNa}$ y sin agente porógeno. Allí puede verse claramente la morfología de las partículas que componen estos materiales. La micrografía de la sílice $\mathrm{B}-\mathrm{MSiNa}$, producida con metasilicato de sodio sin agente porógeno muestra que el esta compuesto por la aglomeración de partículas regulares cúbicas de aproximadamente $1 \mu \mathrm{m}$. 
(a)

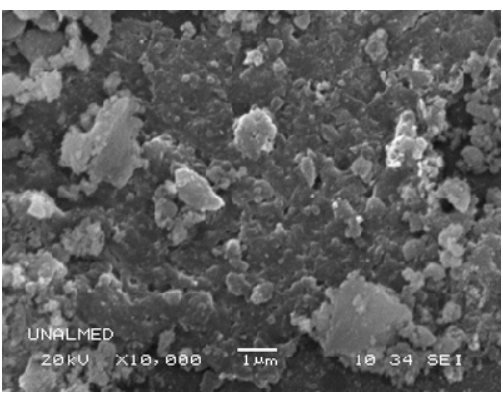

(b)

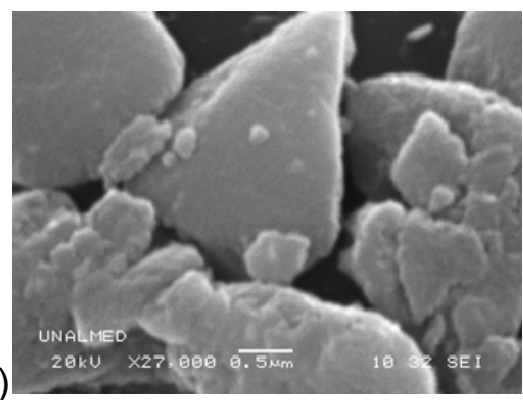

(c)

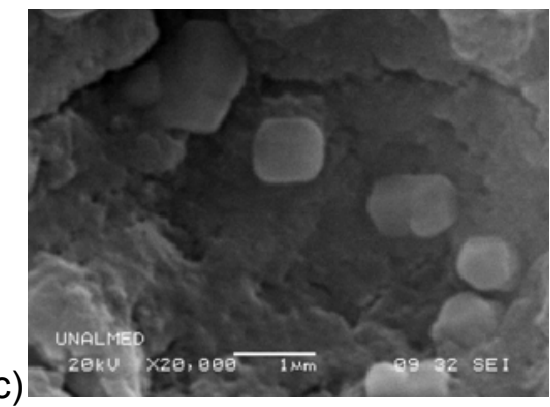

Fig. 2: Micrografías SEM de las sílices (a) TEG2, (b) MEG2, y (c) B-MSiNa.

Las sílices con estas características morfológicas no tendrían aplicación como fases estacionarias en cromatografía líquida de alta definición (HPLC) o como material abrasivo para el tratamiento superficial químico-mecánico acabado espejo, donde la forma de las partículas asume un rol muy importante. Sin embargo, en aplicaciones donde esta característica es de baja importancia y el área superficial y demás características porosas son dominantes, como es el caso de su uso como soporte de catalizadores para formar catalizadores heterogéneos, estas sílices producidas con los porógenos en mención cobran valor y se hace interesante investigar su aplicación en catálisis heterogénea. Las características texturales de las sílices producidas se muestran en la tabla 2.

Tabla 2: Características porosas de las sílices

\begin{tabular}{cccc}
\hline Síntesis & Área BET $\left[\mathbf{m}^{2} / \mathbf{g}\right]$ & $\begin{array}{c}\text { Volumen total de } \\
\text { poro }\left[\mathbf{c m}^{\mathbf{3}} / \mathbf{g}\right]\end{array}$ & $\begin{array}{c}\text { Diámetro de poro } \\
{[\AA]}\end{array}$ \\
\hline TEG 1 & 200.05 & 0.62 & 68.5 \\
TEG 2 & 396.2 & 0.83 & 57.8 \\
TEG 3 & 727.4 & 0.50 & 46.2 \\
B-TEOS & 119.9 & 0.80 & 114.4 \\
MEG 1 & 393.2 & 0.86 & 66.7 \\
MEG 2 & 368.5 & 0.75 & 79.8 \\
MEG 3 & 279.1 & 0.35 & 47.3 \\
B-MSiNa & 191.7 & 0.76 & 95.9 \\
\hline
\end{tabular}

Las sílices preparadas con TEOS muestran una marcada tendencia a incrementar el área superficial cuando se incrementa la relación molar Si:tensoactivo, es decir, cuando se aumenta la cantidad de precursor de sílice. Para los casos donde se uso metasilicato de sodio ocurre todo lo contrario, el área superficial disminuye a medida que se incrementa esta relación molar.

El volumen total de poros, no muestra una tendencia clara en ninguno de los casos y esto se debe a que la técnica usada para medir esta variable incluye tanto poros intra-particulares como poros extraparticulares y de esta manera se incluye en la medida los micro y mesoporos en todo el sólido. Las sílices B-TEOS y B-MSiNa obtenidas sin agente porógeno muestran un valor bastante alto según puede apreciarse en la Tabla 2. Esta medida incluye meso- y macroporos tanto intra- como extraparticulares; pese a este valor alto de volumen total de poro su área superficial BET es baja y su curva de distribución de tamaños de poro (Figura 3) es bastante ancha y achatada, lo que confirma la presencia de mesoporos extraparticulares los cuales se manifiestan con valores de volúmenes totales de poros altos. Este tipo de mesoporos extraparticulares se generan por el acomodamiento libre de las partículas sólidas, son inestables y tienden a desaparecer cuando el material es sometido a esfuerzos de compresión, como por ejemplo en procesos de empastillado para su uso en catálisis heterogénea.

De acuerdo con los valores reportados en la tabla 2 para el diámetro de poro, los poros de todas las sílices obtenidas están dentro del rango meso (20 y $500 \AA$ Å). Las sílices preparadas con TEOS muestran una tendencia a disminuir su tamaño de poro a medida que se incrementa la relación molar Si:tensoactivo. Para los casos donde las sílices fueron preparadas con metasilicato de sodio no se 
observa una tendencia definida, lo que si es claro, es que las sílices que fueron preparadas sin tensoactivo, sílices B-TEOS y B-MSiNa, presentan los valores más altos para el diámetro de poro. Estos resultados junto con las curvas de distribución de tamaños de poros y sus áreas superficiales son fuertes evidencias de que la porosidad en este material es de tipo extra-particular.

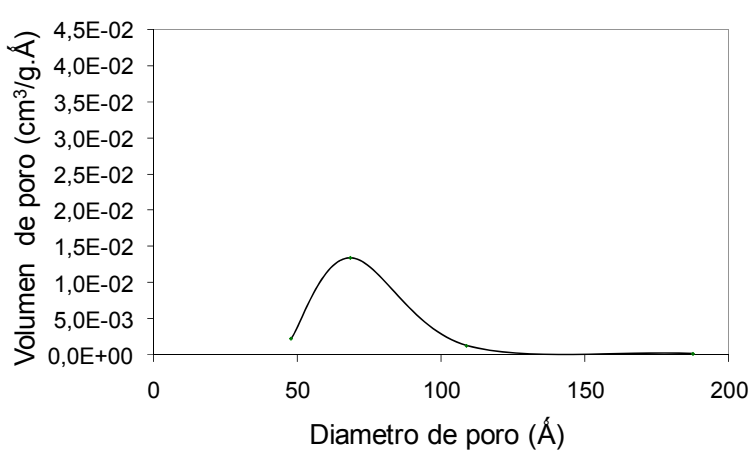

(a)

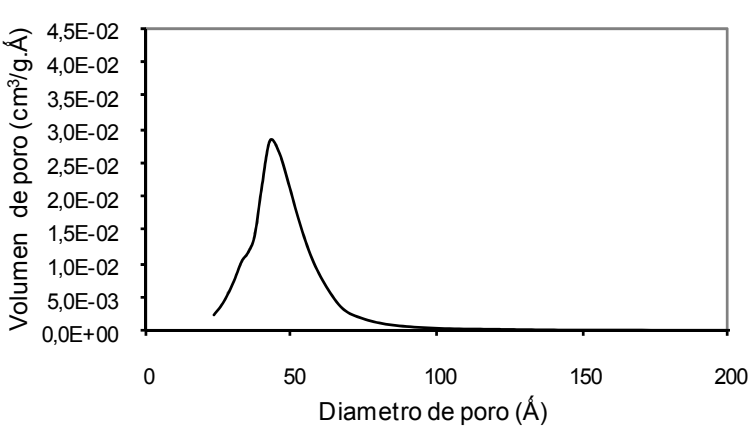

(c)

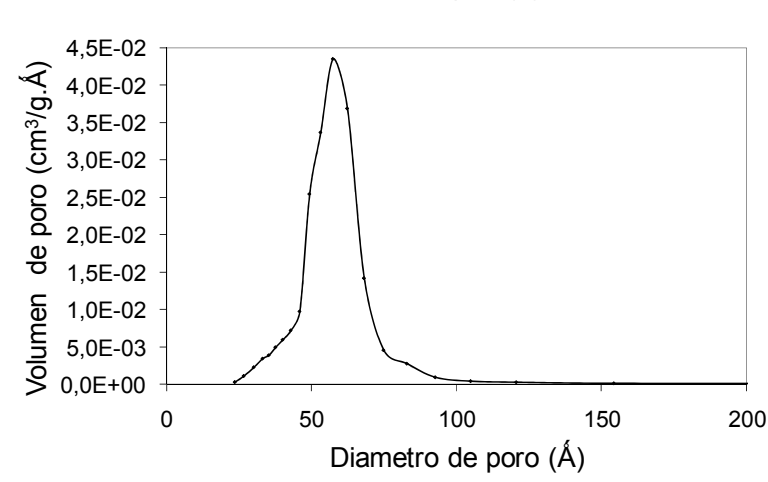

(e)

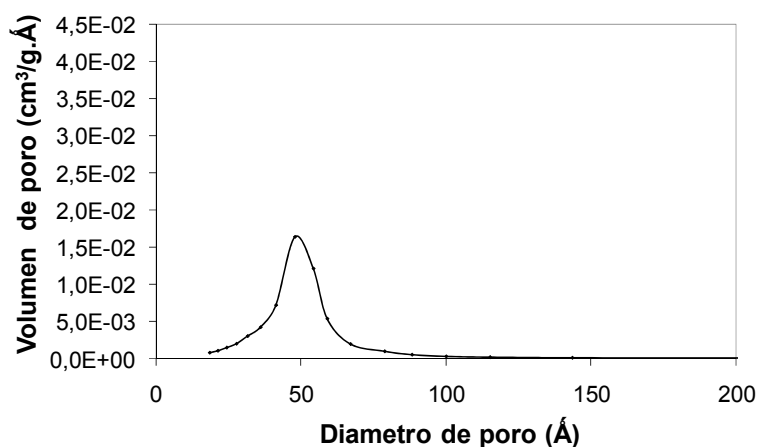

(g)

(d)

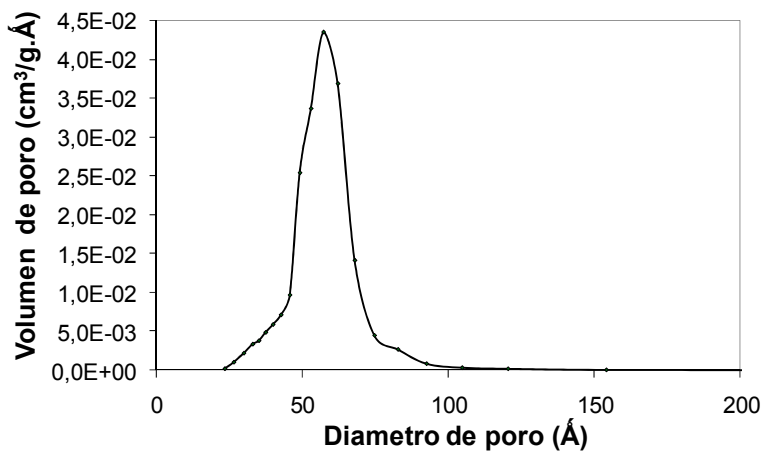

(b)
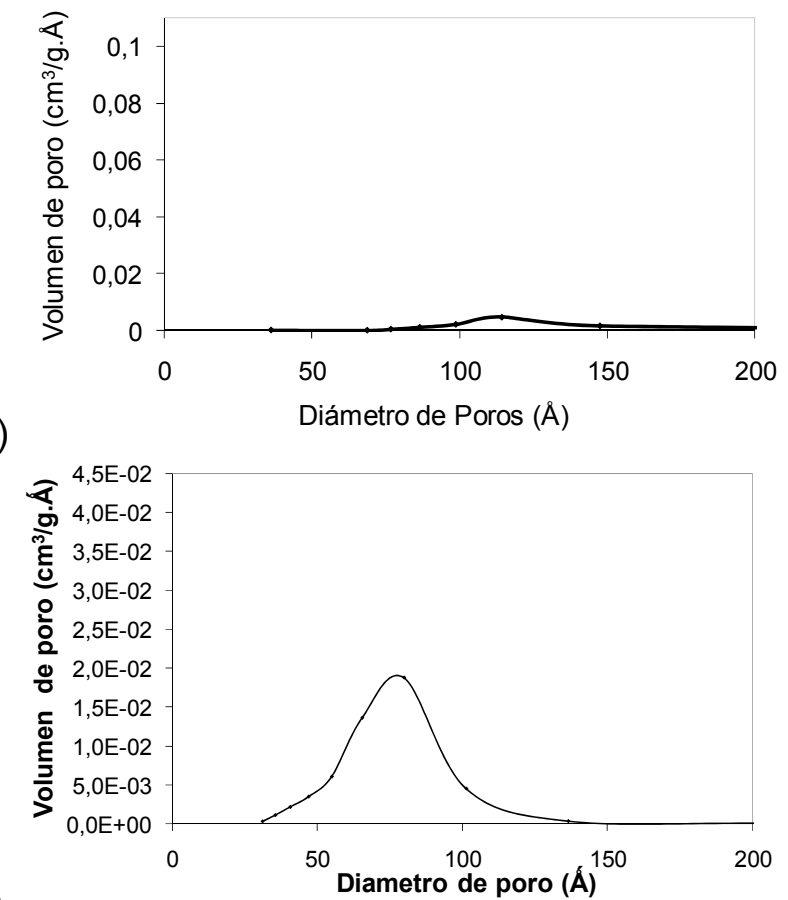

(f)

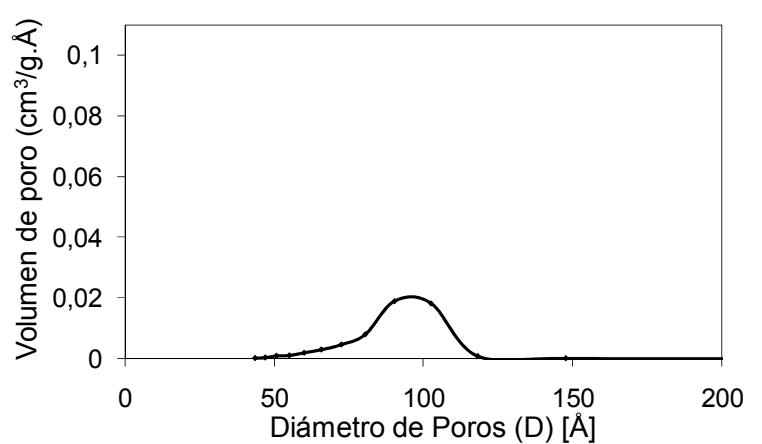

(h)

Fig. 3: Distribuciones de tamaños de poros determinadas con $\mathrm{dV} / \mathrm{dD}$ del método BJH para las sílices producidas con TEOS: (a) TEG1, (b) TEG2, (c) TEG3, (d) B-TEOS, (e) MEG1, (f) MEG2, (g) MEG3, (h) B-MSiNa.

En la figura 3 se muestran las curvas de distribución de tamaños de poro de las sílices que fueron preparadas con TEOS. En ellas puede verse que no hay una tendencia claramente definida que relacione la homogeneidad en el tamaño de los poros con la concentración de precursor de silicio. 
Las curvas de las sílices TEG1 y B-TEOS, son anchas y achatadas, mientras que las correspondientes a las sílices TEG2 y TEG3 son más agudas. A pesar de que la sílice TEG3 fue sintetizada con una concentración de silicato mayor que la sílice TEG2, esta última posee poros más homogéneos en diámetro, lo cual es ventajoso en proceso de separación y adsorción de moléculas en función de su tamaño. Las distribuciones de tamaños de poro de las sílices sintetizadas con metasilicato de sodio (MSiNa), tienden a ser menos homogéneas a medida que se incrementa la cantidad de precursor de silicio. La sílice MEG1 exhibe una curva alta y aguda lo cual indica la formación de poros muy homogéneos en diámetro.

En general, las sílices con las mejores características superficiales fueron aquellas sintetizadas con TEOS. Esto se debe a que las síntesis con MSiNa presentan una fortaleza iónica superior, de tal forma que tan elevada presencia de electrolitos sodio $\left(\mathrm{Na}^{+}\right)$y cloro $\left(\mathrm{Cl}^{-}\right)$hace que la cinética de policondensación sea más rápida y que las los grupos polares del ácido silícico (-Si-OH) tengan mayor afinidad con las moléculas polares del solvente (agua) que con el porógeno. La presencia de sodio en el medio de reacción hace que los puentes de hidrogeno que mantienen débilmente unidas las moléculas de ácido silícico con las micelas del tensoactivo no iónico se rompan, haciendo que menor cantidad de este sea incorporado a la estructura de la sílice formada.

\section{CONCLUSIONES}

El método de síntesis de sílices mesoporosas desarrollado que involucra el uso monoesterato de glicerol como agente porógeno, conduce a la formación de un material silíceo meso-estructurado con características superficiales adecuadas para una potencial aplicación en catálisis heterogénea, gracias a su alta área superficial, adecuados volumen y tamaño de poros; además, este porógeno conducen a la formación de aglomerados de partículas completamente amorfas e irregulares, de tamaños variados y con características porosas diversas según la cantidad y el tipo de precursor de silicio usado.

Las sílices producidas con alcóxido de silicio (tetraetilortosilicato TEOS) exhiben mejores características mesoporosas, mayores áreas superficiales BET, mayor volumen total de poros y distribuciones de tamaños de poro mas homogéneas, que las producidas con metasilicato de sodio.

El proceso de síntesis que condujo a la producción de sílices con las mejores características mesoporosas, haciendo claridad que áreas superficiales entre 500 a $1000 \mathrm{~m}^{2} / \mathrm{g}$ son consideradas altas según las referencias consultadas (Pérez-Pariente, 2006), es la síntesis TEG3 con un área superficial BET de $727.4 \mathrm{~m}^{2} / \mathrm{g}$, volumen total de poros de $0.50 \mathrm{~cm}^{3} / \mathrm{g}$ y estrecha distribución de tamaños de poro alrededor de $46.2 \AA$ Á. Las características de ésta sílice para catálisis heterogénea son excelentes.

\section{AGRADECIMIENTOS}

Al grupo de investigación Procesos Fisicoquímicos Aplicados (PFA), al CODI, a la Universidad de Antioquia y a COLCIENCIAS por su financiación.

\section{REFERENCIAS}

Aramendia M.A. y otros cuatro autores; Poly(ethylene oxide)-based surfactants as templates for the synthesis of mesoporous silica materials. J. Colloid Interface Sci: 269, 394-402 (2004).

Biswas K. y otros cuatro autores; Synthesis of MSU-1 silica particles with spherical morphology. Stud. Surf. Sci. Catal: 165 (2007) 587-590.

Biswas K., J.C. Ray, J. Choi y W. Ahn; Morphology control of MSU-1 silica particles. J. Non-Cryst. Solids: 354, 1-9 (2008).

Chemical land 21, GLYCEROL MONOSTEARATE (2006). http://chemicalland21.com/lifescience/ foco/GLYCEROL\%20MONOSTEARATE.htm. Acceso: 31 de Julio de 2008. 
Guth J.L., M. Mesa y L. Sierra; Formation mechanism of SBA-3, SBA-15 and SBA-16 type mesoporous silica in acidic solutions. Stud. Surf. Sci. Catal.: 170, Part 2, 1850-1855 (2007).

Jin Z., X. Wang y X. Cui; Acidity-dependent mesostructure transformation of highly ordered mesoporous silica materials during a two-step synthesis. J. Non-Cryst. Solids: 353, 2507-2514 (2007a).

Jin Z., X. Wang y X. Cui; A two-step route to synthesis of small-pored and thick-walled SBA-16-type mesoporous silica under mildly acidic conditions. J. Colloid Interface Sci: 307, 158-165 (2007b).

Leonard A. y otros cuatro autores; Chemistry of silica at different concentrations of non-ionic surfactant solutions: effect of $\mathrm{pH}$ of the synthesis gel on the preparation of mesoporous silicas. Microporous and Mesoporous Materials: 63, 59-73 (2003).

Liu J., Q. Yang, X.S. Zhao y L. Zhang; Pore size control of mesoporous silicas from mixtures of sodium silicate and TEOS. Micropor. Mesopor. Mater: 106, 62-67 (2007).

Mesa M., L. Sierra y J.-L. Guth; Contribution to the study of the formation mechanism of mesoporous silica type SBA-3, Micropor. Mesopor. Mater: 102, 70-79 (2007).

Mesa M., L. Sierra y J.-L. Guth; Contribution to the study of the formation mechanism of mesoporous SBA-15 and SBA-16 type silica particles in aqueous acid solutions. Micropor. Mesopor. Mater: 112, 338-350 (2008).

Meynen V., P. Cool y E.F. Vansant; Synthesis of siliceous materials with micro- and mesoporosity. Micropor. Mesopor. Mater: 104, 26-38 (2007).

Pérez-Pariente J., Capitulo I, Materiales mesoporosos de oxido de silicio. [Monografía XIX Liberación de fármacos en matrices biocerámicas: avances y perspectivas]. Instituto de España, Real academia nacional de farmacia, 39-64 (2006).

Prouzet E. y C. Boissière; A review on the synthesis, structure and applications in separation processes of mesoporous MSU-X silica obtained with the two-step process. C.R. Chimie: 8, 579-596 (2005).

Sierra L., B. López y J.L. Guth; Preparation of mesoporous silica particles with controlled morphology from sodium silicate solutions and a non-ionic surfactant at $\mathrm{pH}$ values between 2 and 6 . Micropor. Mesopor. Mater.: 39, 519-527 (2000).

Sierra L., S. Valange, J. Barrault y J.-L. Guth; Templating behavior of a triblock copolymer surfactant with very long hydrophilic PEO chains (PEO140PPO39PEO140) for the synthesis of cubic mesoporous silica with large cage-like cavities. Microp. Mesop. Mat.: 113, 352-361 (2008).

Vacassy R. y otros cuatro autores; Synthesis of Microporous Silica Spheres. Colloid Interface Sci: 227 (2000) 302-315.

Venkatathri N.; Synthesis of mesoporous silica nanosphere using different templates. Solid State Communications: 143, 493-497 (2007).

Wang Q. y D.F. Shantz; Ordered mesoporous silica-based inorganic nanocomposites. J. Solid State Chem.: 181, 659-1669 (2008). 